\title{
Histo-Projections TOF Data Non-Rigid Motion Estimation and Correction
}

This paper was downloaded from TechRxiv (https://www.techrxiv.org).

\section{LICENSE}

CC BY 4.0

SUBMISSION DATE / POSTED DATE

$16-01-2021 / 20-01-2021$

\section{CITATION}

Panin, Vladimir (2021): Histo-Projections TOF Data Non-Rigid Motion Estimation and Correction. TechRxiv. Preprint. https://doi.org/10.36227/techrxiv.13588991.v1

DOI

10.36227/techrxiv.13588991.v1 


\title{
Histo-Projections TOF Data Non-Rigid Motion Estimation and Correction
}

\author{
Vladimir Y. Panin, Member, IEEE, Eva E. Panin, Deepak Bharkhada, Member, IEEE and William Whiteley, \\ Member, IEEE
}

\begin{abstract}
With advancements in PET technology, scanners with a very good time-of-flight (TOF) resolution became available. In addition, scanners with long axial extent are now commercialized. Although raw data sizes increase significantly with such scanners, good TOF resolution allows substantial data compression without any loss of spatial resolution: for example, the histo-projections with relatively coarse sampling in the TOF direction in the Siemens Biograph Vision and Vision Quadra PET/CT scanners.

The patient motion during the scan is unavoidable. The pattern caused by breathing may result in a relatively large displacement of organs and the consequent blurring of clinically relevant features in regions affected by the motion. As was shown in our previous work, non-rigid motion correction can be performed in the quasi-image space of histo-projections. The TOF locality property can be used to locally perform motion correction; that is, the approximation of motion as locally rigid on a scale of TOF resolution. In this work, we investigate motion estimation in the TOF data space from histo-images by histogramming the data into a 3D array consistent (in geometry and size) with the reconstruction image. The histo-image estimated motion is then used in histo-projections motion correction.

Initial results using an XCAT digital phantom showed that the presented methodology accommodates for changes in non-rigid body movements for a typical pattern of patient motion. The patient scan on newly developed Biograph Vision Quadra PET/CT scanner was used in validation of presented motion correction.
\end{abstract}

\section{INTRODUCTION}

$\mathrm{P}$ ET acquisition requires long scan times, making patient motion during the scan unavoidable. For example, the pattern caused by breathing may result in a relatively large displacement of organs and the consequent blurring of clinically relevant features in regions affected by the motion [1].

The breathing pattern can be monitored either by external devices [2] or by tracing the movement of objects in the list mode file [3]. Once the breathing pattern is established, data can be separated into gates according to the phase or amplitude of respiration. Each gate represents a particular (quasi) frozen phase of motion. The gate with minimally integrated motion can be chosen to reconstruct the PET image [4]. While the motion artifact is suppressed, image quality suffers from a greater amount of noise. To achieve motion

Manuscript received December 20, 2020.

V.Y. Panin, D. Bharkhada and W. Whiteley are with Siemens Medical Solutions USA, Molecular Imaging, Knoxville, TN 37932 USA (telephone: 865-368-7086, e-mail: vladimir.panin @siemenes-healthineers.com).

E.E. Panin is with Webb School of Knoxville and Siemens research fellowship program, Knoxville, TN 37923 USA.

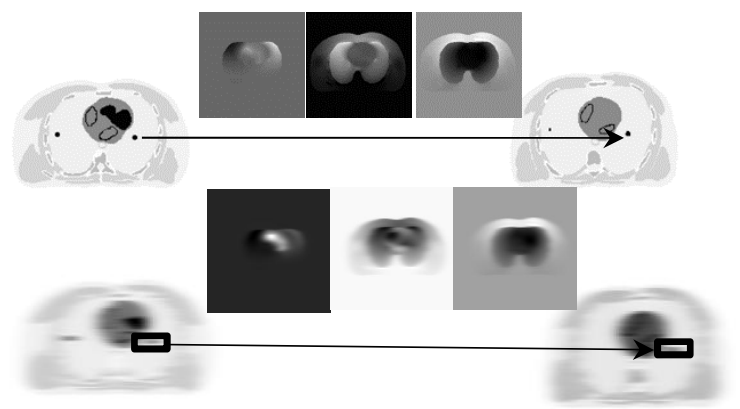

Fig. 1. Schematic presentation of histo-projections MC. The top scheme shows image-based MC, where the voxel of the target image is "pulling" value from the other gate image. Three images above the arrow are $x, y$, and $z$ motion images. The bottom scheme shows a similar procedure, now in the TOF domain. Image and MVF images were blurred and down-sampled according to the TOF model. TOF blurring was applied in the horizontal direction in this case.

artifact suppression and approach maximum signal-to-noise ratio (SNR), each gate's data can be individually reconstructed and registered to one target (typically that of the chosen gate) image. The aligned images are averaged at the end [5]. A better approach is to reconstruct one image from all available data through the incorporation of the field of motion into the reconstruction process [6-8]. However, this approach is fairly computationally extensive and also requires assessment of the motion phase for correction factors such as attenuation.

Estimating the field of motion is a challenging task since the non-rigid model consists of a displacement vector per each image voxel. This is an underdetermined problem, and additional constraints such as field of motion spatial smoothness are typically exploited [9].

Compressed TOF data, either histo-projections [10] or histoimages [11], can be considered as quasi-images. Therefore, non-rigid motion correction can be performed in this quasiimage space. In other words, one can use the TOF locality property to locally perform motion correction (approximating it as locally rigid). Fig.1 shows the principle of motion correction (MC) in histo-projections from our previous work [12]. There, we investigated motion correction in the TOF data space, assuming that the field of motion is known. This motion correction will be approximate in nature, since fine sampling in the TOF direction is not preserved in histoprojections. Nevertheless, the approach can be justified by the fact that the motion field is difficult to precisely estimate at high resolutions. The benefit of motion correction in the TOF projection space is that only one data set, corrected for motion, needs to be reconstructed to achieve higher SNR. The histo- 
projections are the data format of the Siemens Biograph Vision scanner [13]. The good TOF resolution, achieved in this type scanners, allows for practical considerations of MC in TOF data quasi-image space.

In current work we concentrate on the task of motion estimation from TOF data. The most appealing approach is combining all quasi-images, corresponding to various projection views and tilts, into a single histo-image. This reduces the problem of sensitivity to noise. In addition to this, traditional motion estimations are also performed on a single reconstructed image. In a histo-image the data are histogrammed into a 3D array consistent (in geometry and voxel size) with the reconstructed image. The single histoimage concept is used indirectly in motion detection (through Centroid of Distribution, COD) [14]. While a histo-image is a blurred version of a true activity image, the motion vector field (MFV) is typically estimated on effectively downsized images.

\section{METHODS}

\section{A. Single Histo-Image}

The histo-image is formed according to Most Likely Annihilation Position where the histogrammer stores the events into the image voxels where they were most likely emitted from. It can be considered as the simplest form of non-statistical real-time reconstruction in case of perfect TOF resolution. In this work, the histo-image is time dependent: it is gated according to an underlying motion trigger, respiratory amplitude signal. Correction (attenuation, scatter and normalization) factors may be computed in the histo-image format straightforwardly if needed.

\section{B. Optical Flow}

The Lucas-Kanade method [15] is used to estimate the MVF. This method assumes that MVF is the same in the neighborhood of particular voxel, and this is favorable assumption in the case of blurred histo-images. The optical flow methods in general work with relatively small motion, which is a reasonable assumption in respiratory motion given enough sampling of the respiratory cycle. On the other hand, LK optical flow is a purely local method: it cannot provide flow information in the interior of uniform regions of the image.

In the work below, optical flow MVF estimation was applied to adjacent gates only due to better performance in the case of small motion. The MVF between distant gates was obtained by combining MVF of intermediate gates. For example, MVF between gate 1 and 4 is a combination of MVF between gates 1 and 2, 2 and 3, and 3 and 4 .

\section{Computer Simulations}

An XCAT phantom [16] was used in the computer simulations. Eight "respiratory gated" images were produced along with motion displacement fields. A significant respiratory motion of about $1.5 \mathrm{~cm}$ in magnitude was simulated in the activity. TOF PET histo-images and histoprojections were created for each activity image using Siemens Biograph Vision scanner [17] specifications. No scatter, attenuation, randoms and normalization were simulated. Six spherical hot spots were inserted in the lung region, and their recovery in this reconstructed image will serve as figure of merit in comparing methods. Poisson noise was added into histo-image, corresponding to noise level in a typical patient scan.

After histo-image MFV estimation, motion correction is performed in histo-projection space [12] and images are reconstructed by OS-EM algorithm, 5 subsets. The gated images were also assembled by the "pull" method using simplest nearest neighbor interpolation, to produce the MC image with true motion. No MC image is produced by simple gated images summation.

\section{Patient Data}

The newly developed Siemens Vision Quadra PET/CT [18] patient data was used in the validation of MFV estimation. The patient of extended colon disease and significant liver respiratory displacement (about $1 \mathrm{~cm}$ ) was chosen. The patient was administrated with $258 \mathrm{MBq}$ of FDG with a $120 \mathrm{~min}$. uptake. Single bed scan was a $4 \mathrm{~min}$ duration. In reconstructions, a maximum ring difference of 85 was used, the current clinical reconstruction protocol. In histo-image formation, a maximum ring difference of 322 (counting detector axial gaps) was used.

The axial component of COD, the first moment of randoms corrected histo-image, serves as a gating signal. Only part of the histo-image, which is significant in axial extend and covers the lung and abdomen regions, was used in order to get a clearer gating signal. The list mode data was partitioned according to gating signal amplitude, and corresponding gated histo-projections were produced along with corresponding fully corrected OSEM reconstructions. The optical flow MVF estimation was performed over the whole image volume. The obtained MFV was applied on gated reconstructions, resulting in a single $\mathrm{MC}$ image. This image was compared against static (no MC) reconstruction, obtained from the original data without motion gating. 

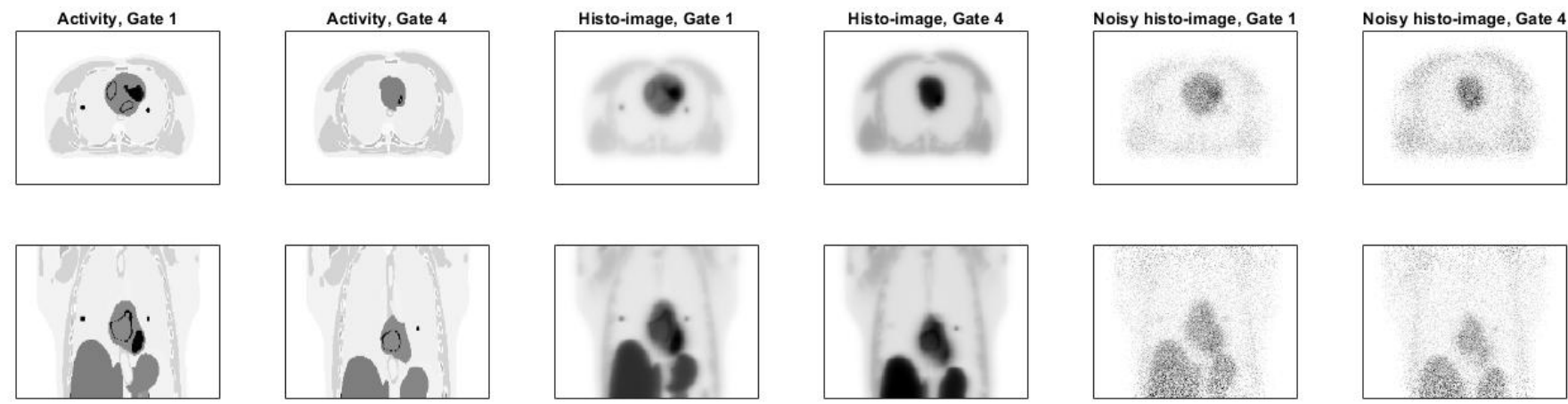

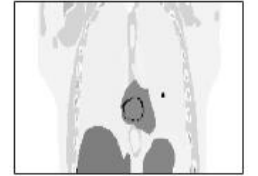

(a)
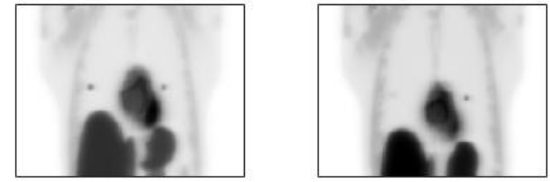

(b)
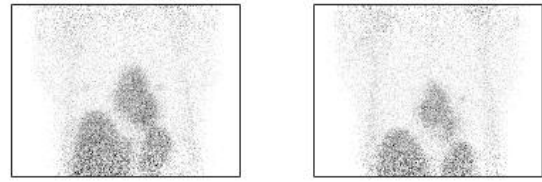

(c)

Fig. 2. Computer Simulations. (a) XCAT activity, (b) noise-free histo-images and (c) Poisson noise added histo-images. Top images are transaxial slices; bottom images are coronal slices. The most distant gate images are shown.

\section{RESULTS}

Fig. 2 presents gated simulated activity, the most distant gates, and corresponding histo-images. The simulated $215 \mathrm{ps}$ TOF resolution produces noticeable blurring effect in latter images. Poisson nose was added to histo-images of fig. 2(b), resulting in fig. 2(c) images.

Fig. 3 presents MVF true distribution and its estimation from histo-images. The axial component is shown, representing the axis of major displacement. While this motion is close to true distribution, it is blockier (a relatively large neighborhood size was used). Nevertheless, even noisy data estimation was approximately correct in the displacement value of spherical lesions. As expected, motion was "absent" in uniform regions. In the case of nosy histo-images, additional $4 \mathrm{~mm}$ Gaussian filter smoothing was necessary to get an estimation of similar magnitude (otherwise low magnitude MVF was estimated, not shown).

Fig. 4 represents reconstruction from histo-projections with noisy free histo-images, fig. 4 (a), and noisy histo-images, fig. 4(b), MVF estimation. For comparison, images with space MC gate assembly with true MFV, fig. 4(c) and no MC images, fig. 4(d) are provided. Both reconstructions from MC histo-projections retained the main features (blurred in no $\mathrm{MC}$ image) of an $\mathrm{MC}$ image in the heart and liver region. A deeper look is presented by Fig 5. Sphere values recoveries are plotted in all methods. The no MC image results in low recovery, while image space $\mathrm{MC}$ image can serve as a gold standard, though when used with nearest neighbor interpolation results in some recovery loss. The MC reconstructions recoveries are close to the gold standard.

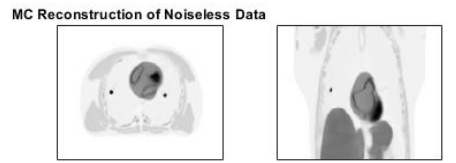

(a)

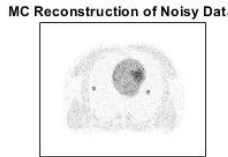

(b)

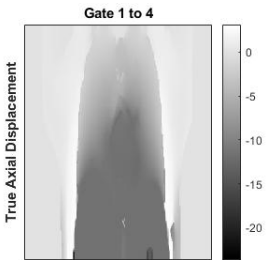

(a)

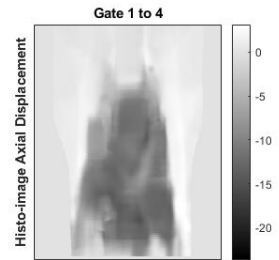

(b)

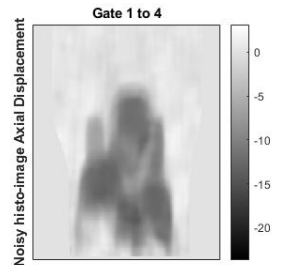

(c)

Fig. 3. Computer simulations. (a) True displacement axial component and its estimation estimated from (b) noise free and (c) noisy histo-images. Displacement is in pixel units.

Noise addition to histo-images led to contrast loss, sometimes significant.

Fig. 6 presents a gating procedure in case of patient data. COD axial component clearly showed the respiratory cycle, with a period of about $5 \mathrm{sec}$. COD time samples were assigned to four gates, based on amplitude of additionally processed COD signal, such as high frequency removal and base line correction. Fig. 7 presents all three components of MVF estimation from histo-images for the case of the most distant gates, 1 and 4 . Finally, Fig. 8 shows a gated histoimage, fig. 8(a) and gated reconstruction, fig. 8(b). Improvement by removing motion blur and retaining the same noise level is apparent in the liver/colon region in comparison between image space $\mathrm{MC}$ reconstruction fig. 8(c) and static no $\mathrm{MC}$ reconstruction fig. 8(d).

\section{DISCUSSION}

The presented work validated histo-image-based motion correction in the case of achievable good TOF resolution. A standard optical flow algorithm was used, and the whole-body

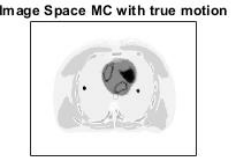

(c)

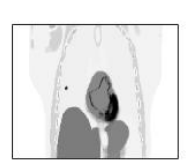

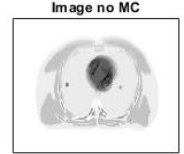

(d)

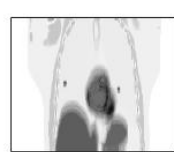

Fig. 4. Computer simulations. Histo-projections MC reconstruction from (a) noise-free data and (b) noisy data. (c) Image space true displacement MC image. (d) no MC image. 

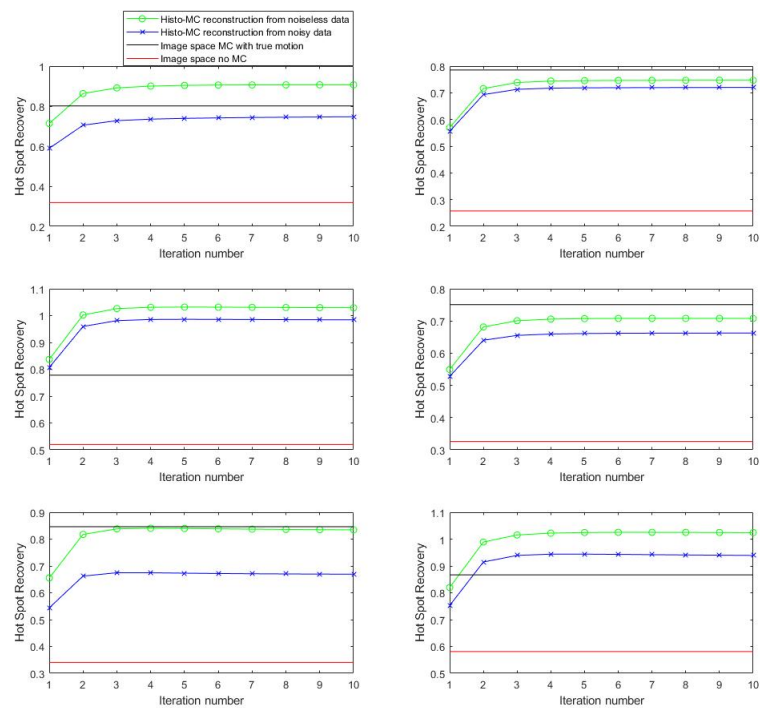

Fig. 5. Computer simulations. Six hot spot recoveries versus iteration number (applied in case of reconstructions). The black curve represents image space $\mathrm{MC}$ with true motion; the red curve represents no $\mathrm{MC}$ image; the green curve represents reconstruction from noise free $\mathrm{MC}$ histo-projections; the blue curve represents reconstruction from noisy MC histo-projections.

study showed that this algorithm is capable of distinguishing respiratory motion in some body parts, such motion in the liver and identifying no motion in the rest of body, such as head. Nevertheless, noise histo-image should be additionally monitored for better estimation of image edges and MVF which will result in additional parameters to tune. As expected, motion was "absent" in uniform regions in computer simulations results. This may not be the issue for typical PET patient study, where the goal is hot spot detection and its improved quantifications. The more complicated registration algorithm can be used to overcome limitation of optical flow method.

The original data format is histo-projection, where azimuthal and polar angle sampling preserves spatial resolution, except in the TOF dimension. Producing single histo-image, the most compressed form in terms of angle sampling, decreased spatial resolution, but it may not affect presented results, since MFV is effectively estimated on a lower sampling grid. An attempt fitting MVF into multiple histo-images should be of interest to see how excessive angle

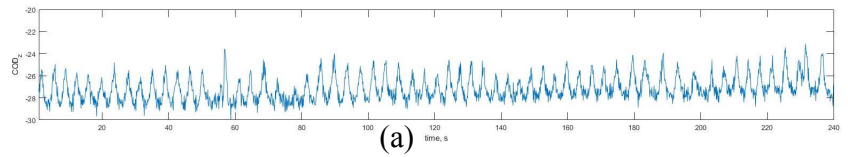

(a)

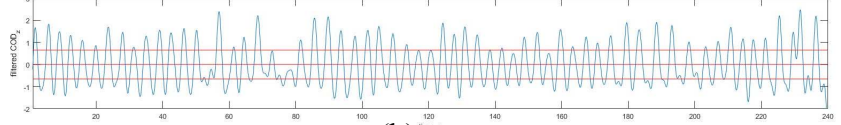

(b)

Fig. 6. Patient data. COD axial component as a gating signal. (a) Original signal; (b) additionally processed signal along with amplitude gating regions.
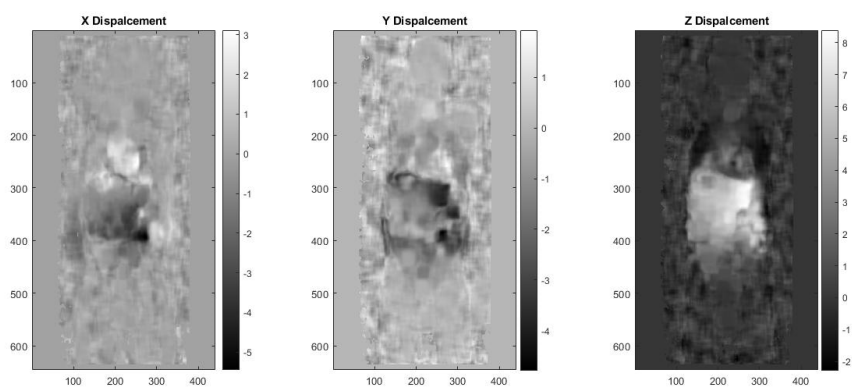

Fig. 7. Patient Data. Displacement estimation components. MVF was estimated inside image support, defined by an attenuation map. Displacement is in pixel units.

view noise problems can be overcome [19].

The MVF was applied in histo-projections in the case of computer simulations, through the technique presented in [12]. This resulted in additional blurring of MVF in the TOF direction of a particular angle sample and this may affect results, since histo-images based MVF estimation is blurred from the beginning. In the case of patient data, MC was performed in an image space. Though this established MC technique is not optimal, it served as validation of motion estimation. Our goal is to perform MC entirely in TOF data space, so additional work, especially in terms of normalization correction, is needed to finalize patient data processing. In the case in patient data, attenuation correction is an open question, since a CT-based attenuation map does not represent gated attenuation maps.

Our investigation of fast PET [20], based on Deep Leaning, where the goal is achieving high quality, not necessary diagnostic, PET images from histo-images in real time is currently undergoing. It will be interesting to understand if additional DL processing of histo-images will result in MVF estimation improvement.

\section{CONCLUSIONS}

The presented methodology performs approximate nonrigid motion correction in TOF quazi-image space. The preliminary results, including whole body imaging on a long axial field-of-view scanner of good TOF resolution, show that, in principle, the MVF can be estimated by the standard optical flow method from a single histo-image.

MVF estimation was found to be sensitive to noise in the histo-images and additional smoothing of noisy histo-image was necessary. The noise can be treated by Deep Learning, for which investigations are ongoing.

\section{ACKNOWLEDGMENT}

The authors would like to thank Bern University Hospital's Inselspital Department of Nuclear Medicine, the first site of Siemens Vision Quadra, for providing patient data. 


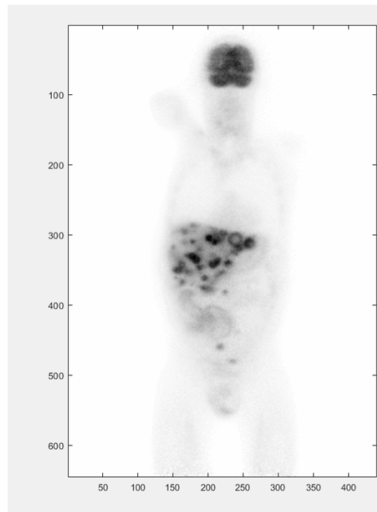

(a)

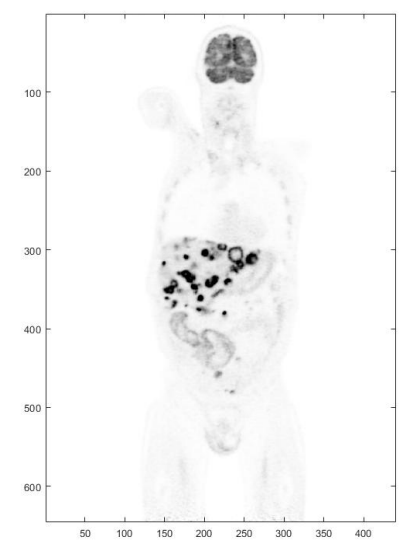

(c)

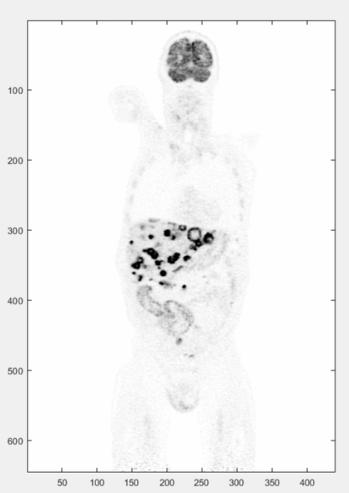

(b)

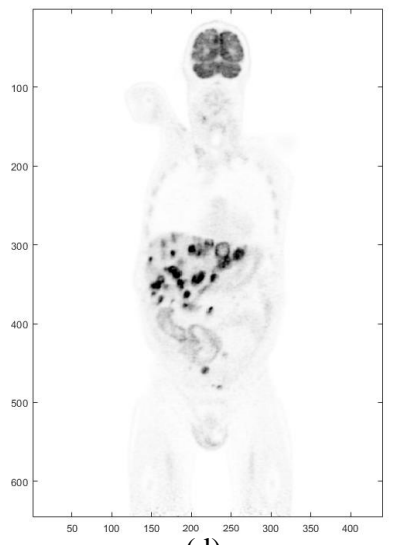

(d)
Fig. 8. Patient Data. (a) gated normalization, attenuation and randoms corrected histo-image; (b) gated fully corrected reconstruction; (c) image space $\mathrm{MC}$ reconstruction; (d) static, no $\mathrm{MC}$, reconstruction.
[9] M. Dawood, F. Büther, X. Jiang, and K. P. Schäfers, "Respiratory motion correction in 3D PET data with advanced optical flow algorithms," IEEE Trans. Med. Img., vol. 27, pp. 1164-1175, 2008.

[10] S. Vandenberghe, M. E. Daube-Witherspoon, R. M. Lewitt, and J. S. Karp, "Fast Reconstruction of 3D TOF PET Data by Axial Rebinning and Transverse Mashing," Phys. Med. Biol., 51, 1603-1621, 2006.

[11] S. Matej, S. Surti, S. Jayanthi, M. E. Daube-Witherspoon, R. M. Lewitt, and J. S. Karp "Efficient 3-D TOF PET reconstruction using viewgrouped histo-images: DIRECT-direct image reconstruction for TOF," IEEE Trans. Med. Img., vol. 28, pp. 739-751, 2009.

[12] V. Y. Panin and H. Bal, "TOF data non-rigid motion correction," 2015 IEEE Nuclear Science Symposium and Medical Imaging Conference.

[13] V. Y. Panin, M. Aykac and I. Hong, "TOF Data Compression on High Time Resolution Clinical Scanner," 2018 IEEE Nuclear Science Symposium and Medical Imaging Conference.

[14] S. Ren, X. Jin, C. Chan, Y. Jian, T. Mulnix, C. Liu, and R. E. Carson, "Data-driven event-by-event respiratory motion correction using TOF PET list-mode centroid of distribution," Phys. Med. Bio., vol. 62, p. 4741, 2017.

[15] B. D. Lucas and T. Kanade, "An iterative image registration technique with an application to stereo vision," Proceedings DARPA Imaging Understanding Workshop, 1981.

[16] W. P. Segars, G. Sturgeon, S. Mendonca, J. Grimes, and B. M. W. Tsui, "4D XCAT phantom for multimodality imaging research," Med. Phys., vol. 37, pp. 4902-4915, 2010.

[17] J. J. van Sluis, J. de Jong, J. Schaar, W. Noordzij, P. van Snick, R. Dierckx, R. Borra, A. Willemsen, and R. Boellaard, "Performance characteristics of the digital Biograph Vision PET/CT system," J. Nucl. Med., vol. 60, pp. 1031-1036, 2019.

[18] S. B. Siegel, M. Aykac, H. Bal, B. Bendriem, D. Bharkhada, J. Cabello, L. A. Eriksson, V. Panin, H. Rothfuss, M. Conti, "Preliminary Performance of a Prototype, One-Meter Long PET Tomograph," 2020 IEEE Nuclear Science Symposium and Medical Imaging Conference.

[19] Y. Li, J. S. Karp, and S Matej, "Motion Displacement Field Estimation using Time-of-Flight PET Histoimages," 2019 IEEE Nuclear Science Symposium and Medical Imaging Conference.

[20] W. Whiteley, V. Panin, C. Zhou, J. Cabello, D. Bharkhada, and J. Gregor, "FastPET: Near Real-Time PET Reconstruction from HistoImages Using a Neural Network," submitted to IEEE Trans. Rad. Pl. Med. Sci.

\section{REFERENCES}

[1] C. Liu, L. A. Pierce, A. M. Alessio, and P. E. Kinahan, "The impact of respiratory motion on tumor quantification and delineation in static PET/CT imaging," Phys. Med. Bio., vol. 54, p. 7345, 2009.

[2] S. A. Nehmeh, Y. E. Erdi, T. Pan, A. Pevsner, K. E. Rosenzweig, E. Yorke, G. S. Mageras, H. Schoder, P. Vernon, O. Squire, H. Mostafavi, S. M. Larson and J. L. Humm "Four-dimensional (4D) PET/CT imaging of the thorax," Med. Phys., vol. 31, pp. 3179-3186, 2004.

[3] F. Büther, M. Dawood, L. Stegger, F. Wübbeling, M. Schäfers, O. Schober, and K. P. Schäfers "List mode-driven cardiac and respiratory gating in PET," J. Nucl. Med., vol. 50, pp. 674-681, 2009.

[4] W. van Elmpt, J. Hamill, J. Jones, D. De Ruysscher, P. Lambin, and M. Öllers, "Optimal gating compared to 3D and 4D PET reconstruction for characterization of lung tumours," Eur. J. Nucl Med. Mol. Imag., vol. 38, pp. 843-855, 2014.

[5] Y. Picard and C. J. Thompson, "Motion correction of PET images using multiple acquisition frames," IEEE Trans. Med. Img., vol. 16, pp. 137144, 1997.

[6] T. Li, B. Thorndyke, E. Schreibmann, Y. Yang, and L. Xing, "Modelbased image reconstruction for four-dimensional PET," Med. Phys., vol. 33, pp. 1288-1298, 2006.

[7] F. Qiao, T. Pan, J. W. Clark, and O. R. Mawlawi, "A motion incorporated reconstruction method for gated PET studies," Phys. Med. Bio., vol. 51, pp. 3769-3783, 2006.

[8] F. Lamare, M. J. L. Carbayo, T. Cresson, G. Kontaxakis, A. Santos, C. C. Le Rest, A. J. Reader, and D. Visvikis, "List-mode-based reconstruction for respiratory motion correction in PET using non-rigid body transformations," Phys. Med. Bio., vol. 52, p. 5187, 2007. 